\section{A Simple Lecture Experiment with Radium Rays.}

WhILST preparing some experiments for a lecture on this matter, I found a very simple device to demonstrate the important fact that radium rays are very easily transmitted through a high vacuum; and $I$ am not aware that it has been published before in this way. I had at my disposa the strongly acting compound of radium bromide which is prepared at Brunswick, in Germany; io mgr. were enclosed in a small box of ebonite with a mica cover having a diameter of $20 \mathrm{~mm}$. This was put down in a Dewar's tube with vacuum jacket, as is commonly used in experiments with liquid air, and held in place by a stopper of cotton wool. The tube was then turned upside down in a little dish with some mercury, so as to obtain a perfectly enclosed space, and the radium rays could only get out by the vacuum walls or through a thick layer of mercury by taking enough of this dense liquid the escape may be stopped altogether. Putting now a charged sensitive gold leaf electroscope at a distance of $5 \mathrm{~cm}$. from the tube, a leakage instantly sets in, so as to cause the instrument to be wholly discharged in fifteen seconds. I. also tried a vacuum jacketed tube with silvered walls, but though this affords much better protection against the heat rays, I did not detect any considerable difference with regard to the former experiment; the discharge was almost as quick, demonstrating that radium rays are not reflected to an appreciable amount. Even when the radium bromide was put into a large Dewar's silvered balloon of 5 litres capacity, wrapped in cotton wool, and enclosed in a wooden case, in which liquid air would be preserved during more than a fortnight, the charged electroscope came to zero in half a minute when it was placed very near to it. The experiments are effective and easily arranged.

'The Hague, November 20.

\section{Nuclei and Ions}

IT is perhaps ungracious to reply to a review. I appreciate very fully that in cases of papers like mine, which take an isolated position and are written by a man who is not infallible, the task. of the reviewer is burdensome enough. But Mr. C. T. R. Wilson's summary of several years of my work (October 8, p. 548) seems to me unnecessarily captious, and I am obliged to answer in selfdefence.

I will not quarrel with Mr. Wilson about the titles of $\mathrm{m}_{j}$ papers, or about references to my first paper ("Experiments with Ionised Air"). I have had occasion to come back to it myself since (Amer. Jour. Sci., xv., 105; ibid. 217 ), and shall presumably do so again.

Turning to the second paper ("Structure of the Nucleus "), the impression given is that my first chapter is superfiuous. The particular direction in which $\mathrm{Mr}$. Wilson thinks it superfluous, i.e. the determination of reciprocal relations in the number of ions and nuclei arising is any process, I consider of special importance, as I shall explain beiow. Apart from this, the gist of the chapter is the (to me) very interesting result that phosphorus as a nucleator suddenly bursts forth into maximum activity at about $13^{\circ}$. The smoke at higher temperatures is a de gradation. If I had made these experiments earlier I should not have drawn the comparison between the number of nuclei and the number of ions which Mr. Wilson impales. Recently (Amer. Jour. Sci., xv., 217) I have departed widely from this early result.

With regard to my work on coronas, I had hoped that any rational attempt at systematisation would at least be tolerated. It was something, I thought, to plough through so bewildering a display and to get the general lay of the land in that deceptive colour territory, to distinguish sharply between the axial and the coronal colours, to ascertain that even in the former case the particles are large in comparison with the wave-length of light. So far as I know a discrimination of the evidence obtainable from the steam jet and the condensation chamber has thus for the first time been given. Mr. Wilson, however, has no encouragement. He gravely doubts "whether the method can be made a trustworthy one." Unfortunately I did not know this, for I have since ventured to repeat the whole work (Amer. Jour. Sci., xvi., 325, and a forthcoming paper in Boitzmann's "Jubelband"), with corrections of method No. I 779, VOL. 69] and calculations, obtaining suggestive periodic variations of the coronal apertures for a given colour and the sizes of the cloud particles. I have recently succeeded in catching, holding, and approximately measuring under the microscope the particles of the finest fog (beyond the largest green-biue-purple corona). Again, in a year's continuous observation by my coronal method of the atmospheric nucleation of Providence (lest this lead to " misconception," let me say that no theological bearing is implied), I have found the data useful (Physical Review, xvi., 193; xvii. 233).

My interpretation of the experiments on the diffusion of the nucleus is in error, but I have long since corrected it (fully in Amer. Jour. Sci., June, p. 472 , briefly in Drude's Annalen, August, p. I I44). Hence I do not find $\mathrm{Mr}$. Wilson's belated comments particularly helpful. I was so fully convinced that the excessively slow diffusions observed could only be due to the motion of nuclei that I failed to see that the small coefficients of the hydrocarbon vapours would be virtually accentuated in large degree by the occurrence of diffusion from saturated to somewhat less saturated vapour. But this bad break is not of primary significance in its bearing on my work; the original purpose of these experiments with hydrocarbon vapours, which Mr. Wilson overlooks, was this:-If the ionisation accompanying nucleation is favourable to condensation, it should be particularly so, presumably, in the case of the vapour of an ionising solvent like water. Hence if non-ionising solvents like the hydrocarbons be substituted for water, the absence of effects attributable to ionisation might be discernible. No essential difference was detected.

In the following remarks relative to nuclei produced by shaking liquids, it is astonishing to find a faint note of approval, but Mr. Wilson does not intend that it shall be taken too seriously. "There is nothing new," he hastens t) add, "that nuclei of this kind exist." Verbally, this may be true, but the implication of the whole paragraph is much broader. He does not point out, however, where I may find a prior succinct statement, identical with the view which I give for the persistence of the solutional nucieus.

My "extraordinary hypothesis," as Mr. Wilson calls it, is a critical alternative, put forward to ascertain whether it has been proved that ionisation has an immediate effect on condensation, or whether such condensation is not even now to be regarded as a mere question of the size of the nuclei. The hypothesis should, in the first place, be fairly stated. In any region of intense ionisation there must be a correspondingly marked tendency to synthesis. The nucleus is the stable result of this synthesis. What its structure is to be depends, therefore, primarily on the chemical ingredients of the medium out of which the nucleus is made. Given a definite medium, simple or complex, and one may anticipate a nucleus of definite size and a corresponding supersaturation needed for condensation. My contention is, then, that if nuclei are formed by the $\mathrm{X}$-rays at the anode and the kathode, they are liable to be different, because the ingredients out of which the nuclei are to be compounded are different. If they do not vary in size but merely in number with the intensity of the radiation, this need be no more surprising than that the products of combustion remain the same within a wide range of temperature.

My reasons for this view may best be developed in connection with the case of phosphorus. Mr. Wilson dismisses it by stating. "The answer is simply that the nuclei causing the phosphorus clouds are not free ions like those produced by the X-rays." Let me explain why I fail to grasp the term "free ion." The phosphorus nucleus, as experiment shows, is always a relatively persistent body, while the initial ionisation is to an equal degree characteristically fleeting. Usually before the emanation has been made available for condensation, only a few per cent. of the initial ionisation is left. Meanwhile, the nucleation or condensational activity has suffered no commensurate decline (Physical Review, xvi., 288). It is probable that the whole series of condensations subsequently to be evoked follow in the absence of ionisation.

The case of water nuclei is in this respect almost the same, except that the initial ionisation (I shall venture to call it so, since it discharges both positive and negative 